\title{
Manejo do melasma em mulheres adultas
}

\author{
Management of melasma in adult women \\ Manejo del melasma en mujeres adultas
}

Giovanna Stefanne Lópes Barbosa

ORCID: https://orcid.org/0000-0003-3387-1219

Universidade Federal do Delta do Parnaíba, Brasil

E-mail: gistefanne@gmail.com

Camila Pereira Miranda Costa

ORCID: https://orcid.org/0000-0002-4724-6943

Universidade Federal do Delta do Parnaíba, Brasil

E-mail: camilapcosta319@gmail.com

Mariana Veras Rocha Borges

ORCID: https://orcid.org/0000-0003-2922-4377

Universidade Federal do Delta do Parnaíba, Brasil

E-mail: mariana_vrborges@ hotmail.com

Mary Ângela de Oliveira Canuto Coutinho

ORCID: https://orcid.org/0000-0003-2157-4794

Universidade Federal do Delta do Parnaíba, Brasil

E-mail: maryangelacanuto@gmail.com

Maria Simone Lopes

ORCID: https://orcid.org/0000-0003-2689-3655

Universidade Federal do Delta do Parnaíba, Brasil E-mail: simonemaia617@gmail.com

Bianca Lopes Cacau

ORCID: https://orcid.org/0000-0002-0525-8073

Universidade Federal do Delta do Parnaíba, Brasil

E-mail: bianca.lopescacau1@gmail.com

Marinice Saraiva Attem

ORCID: https://orcid.org/0000-0002-7460-5788 Instituto de Educação Superior Vale do Parnaíba, Brasil

E-mail: mariniceattem@gmail.com

Daniela Winckler Mass

ORCID: https://orcid.org/0000-0003-3655-4040

Universidade Federal do Delta do Parnaíba, Brasil

E-mail: danielamasspi@gmail.com

Breno Serafim Pereira

ORCID: https://orcid.org/0000-0001-5441-9198

Universidade Federal do Delta do Parnaíba, Brasil E-mail: breno-serafim@hotmail.com

Fernando Aguiar Luz

ORCID: https://orcid.org/0000-0002-6625-5941

Universidade Federal do Delta do Parnaíba, Brasil

E-mail: biopele.fernandoluz@ hotmail.com

Ludmilla Figueiredo Vale Fontenelle

ORCID: https://orcid.org/0000-0003-4885-8340

Universidade Federal do Delta do Parnaíba, Brasil E-mail: lufigueiredo@hotmail.com

\section{Resumo}

Objetivo: Descrever os tratamentos disponíveis para melasma em mulheres adultas. Metodologia: Trata-se de uma revisão integrativa acerca dos tratamentos disponíveis para mulheres adultas com melasma, por meio de buscas nas seguintes bases de dados: Medical Literature Analysis and Retrieval System (MEDLINE/PubMed), Scientific Electronic Library Online (SciELO) e Literatura Latino-Americana e do Caribe em Ciências da Saúde (LILACS). Ao todo foram incluídos 23 artigos na presente revisão. Resultados: Após análise dos tratamentos empregados nos trabalhos selecionados, identificou-se cinco categorias de tratamentos, sendo eles: utilização de ácido tranexâmico, tratamentos com microagulhamento, tratamentos com laser, tratamentos com extratos vegetais e outros tratamentos. Conclusão: Este artigo considerou que ao comparar o tratamento tópico com ácido tranexâmico e hidroquinona os resultados são semelhantes, no entanto a segunda opção apresenta mais efeitos adversos. Em pacientes que apresentam recidivas frequentes a associação do microagulhamento contribui com aumento da eficácia do ácido 
tranexâmico, sendo a terapia com laser outra opção de manejo nesses casos. Ademais, as formulações contendo extratos vegetais apresentam melhora das manchas hipercrômicas, assim como outros tratamentos.

Palavras-chave: Melanose; Terapêutica; Dermatologia.

\begin{abstract}
Objective: To describe the treatments available for melasma in adult women. Methodology: This is an integrative review about the treatments available for adult women with melasma, through searches in the following databases: Medical Literature Analysis and Retrieval System (MEDLINE/PubMed), Scientific Electronic Library Online (SciELO) and Literature Latin American and Caribbean Health Sciences (LILACS). Altogether, 23 articles were included in this review. Results: After analyzing the treatments used in the selected works, five categories of treatments were identified, namely: use of tranexamic acid, microneedling treatments, laser treatments, treatments with plant extracts and other treatments. Conclusion: This article considered that when comparing topical treatment with tranexamic acid and hydroquinone the results are similar, however the second option has more adverse effects. In patients with frequent relapses, the association of microneedling contributes to an increase in the effectiveness of tranexamic acid, with laser therapy being another management option in these cases. In addition, formulations containing plant extracts show improvement in hyperchromic spots, as well as other treatments.
\end{abstract}

Keywords: Melanosis; Therapeutics; Dermatology.

\title{
Resumen
}

Objetivo: Describir los tratamientos disponibles para el melasma en mujeres adultas. Metodología: Se trata de una revisión integradora sobre los tratamientos disponibles para mujeres adultas con melasma, mediante búsquedas en las siguientes bases de datos: Medical Literature Analysis and Retrieval System (MEDLINE/PubMed), Scientific Electronic Library Online (SciELO) y Literature Latin American and Caribbean Health Ciencias (LILACS). En total, se incluyeron 23 artículos en esta revisión. Resultados: Tras analizar los tratamientos utilizados en los trabajos seleccionados, se identificaron cinco categorías de tratamientos, a saber: uso de ácido tranexámico, tratamientos de microagujas, tratamientos con láser, tratamientos con extractos de plantas y otros tratamientos. Conclusión: Este artículo consideró que al comparar el tratamiento tópico con ácido tranexámico e hidroquinona los resultados son similares, sin embargo la segunda opción tiene más efectos adversos. En pacientes con recaídas frecuentes, la asociación de microagujas contribuye a incrementar la efectividad del ácido tranexámico, siendo la terapia láser otra opción de manejo en estos casos. Además, las formulaciones que contienen extractos de plantas muestran una mejora en las manchas hipercrómicas, así como otros tratamientos.

Palabras clave: Melanosis; Terapéutica; Dermatología.

\section{Introdução}

O melasma é uma dermatose comum que se manifesta por máculas acastanhadas em áreas expostas à radiação solar, com pigmentação de diferentes intensidades, acometendo, principalmente, mulheres em idade fértil (Costa, Moisés, Cordero, Alves, \& Marmirori, 2010). Acredita-se que a luz solar, a gravidez, os distúrbios da tireoide e a reposição hormonal desempenhem um papel na patogênese, mas a etiologia exata dessa condição ainda não é bem definida (Medeiros, Neves, Moura, \& Medina, 2016).

A terapêutica do melasma é desafiadora e de longa duração, pois o quadro pode ser refratário e os pacientes frequentemente apresentam recidivas, o que pode acarretar problemas de autoestima (Diosti et al., 2012). Atualmente, o tratamento normalmente requer a combinação de diferentes modalidades terapêuticas, como agentes de fotoproteção, clareadores de pele tópicos e orais e procedimentos que auxiliam no controle do quadro clínico (Costa, Sousa, Conradesque, \& Carniel, 2020).

Como forma de avaliar a resposta ao tratamento, são utilizados alguns métodos, como Melasma Area and Severety Index (MASI) e o Melasma Quality of Life Scale (MELASQol). O MASI é utilizado na classificação clínica do melasma, seu cálculo é realizado com base na extensão da lesão nas áreas da face frontal (F), malar direita (MD), malar esquerda (ME) e mentoniana (M) (Magalhães, Borges, Oliveira, \& Neves, 2010). O MELASQol é capaz de avaliar a qualidade de vida dos pacientes acometidos pelo melasma. A partir da análise desses parâmetros, pode-se perceber o impacto que o melasma acarreta na qualidade de vida do paciente, visto que, mesmo em pacientes com melhora nos índices de resposta ao tratamento, o MELASQol ainda pode permanecer baixo, refletindo o incomodo com possíveis manchas persistentes (Hammerschmidt, 
Mattos, Suzuki, Freitas, \& Mukai, 2012).

Além disso, utiliza-se o índice de severidade e área do melasma modificado (mMASI), avaliando-se a pigmentação e a área de envolvimento das quatro regiões da face para acompanhamento das respostas aos tratamentos realizados (Arrowitz et al., 2019).

Nesse contexto, o melasma, como outras patologias que afetam a pele, tem grande repercussão emocional e na autoestima do paciente, podendo desencadear processos de adoecimento mental, como depressão e ansiedade, o que interfere negativamente na qualidade de vida dos pacientes acometidos (Oliveira et al., 2019).

Diante dos diferentes tratamentos empregados no manejo dessa condição e de suas repercussões físicas e emocionais, objetivou-se descrever os tratamentos disponíveis para melasma em mulheres adultas.

\section{Metodologia}

Trata-se de uma revisão integrativa, método que reúne, avalia e sintetiza resultados de pesquisas sobre uma determinada temática. Em virtude da quantidade crescente e da complexidade de informações na área da saúde, tornou-se imprescindível o desenvolvimento de artifícios, no contexto da pesquisa cientificamente embasada, capazes de delimitar etapas metodológicas mais concisas com vistas a propiciar, aos profissionais, uma melhor utilização das evidências elucidadas nos inúmeros estudos (Garcia, Fonseca, Aroni, \& Galvão, 2016). Assim, a revisão integrativa tem sido apontada como uma ferramenta ímpar no campo da saúde, uma vez que condensa os diversos conhecimentos disponíveis sobre determinada temática, direcionando a prática fundamentada em conhecimento científico (Souza, Silva, \& Carvalho, 2010).

Nesse aspecto, esta revisão integrativa se baseia na questão: "Quais os tratamentos disponíveis para mulheres adultas com melasma?". Para buscar resolver tal questão foram realizadas buscas nas seguintes bases de dados: Medical Literature Analysis and Retrieval System (MEDLINE/PubMed), Scientific Electronic Library Online (SciELO) e Literatura LatinoAmericana e do Caribe em Ciências da Saúde (LILACS).

A busca consistiu na combinação, por operadores boleanos, dos descritores (DECS - Descritores em Ciências da Saúde): "melanose", "mulher" e "terapêutica", utilizados nas plataformas SciELO e LILACS, bem como seus correspondentes em língua inglesa (MeSH - Medical Subject Headings) "melasma", "women" e "therapeutics", na busca realizada na MEDLINE/PubMed. Foi considerado o período entre 2010 e 2020, para a busca de artigos.

As estratégias de busca utilizadas foram, respectivamente:

a) Melanose AND mulher AND terapêutica

b) Melasma AND women AND therapeutics

Os critérios de inclusão para a seleção dos artigos foram: artigos em inglês ou português, publicados entre 2010 e 2020, com texto completo disponível e relacionados à pergunta da pesquisa. Como critérios de exclusão, foram elencados: artigos em outros idiomas que não inglês ou português, artigos anteriores a 2010, revisões, artigos baseados em opiniões de especialistas, não disponíveis na íntegra e não relacionados com a pergunta desta pesquisa. Na Figura 1 é possível observar o método utilizado para a seleção dos artigos. 
Figura 1 - Seleção dos artigos para a revisão de literatura.

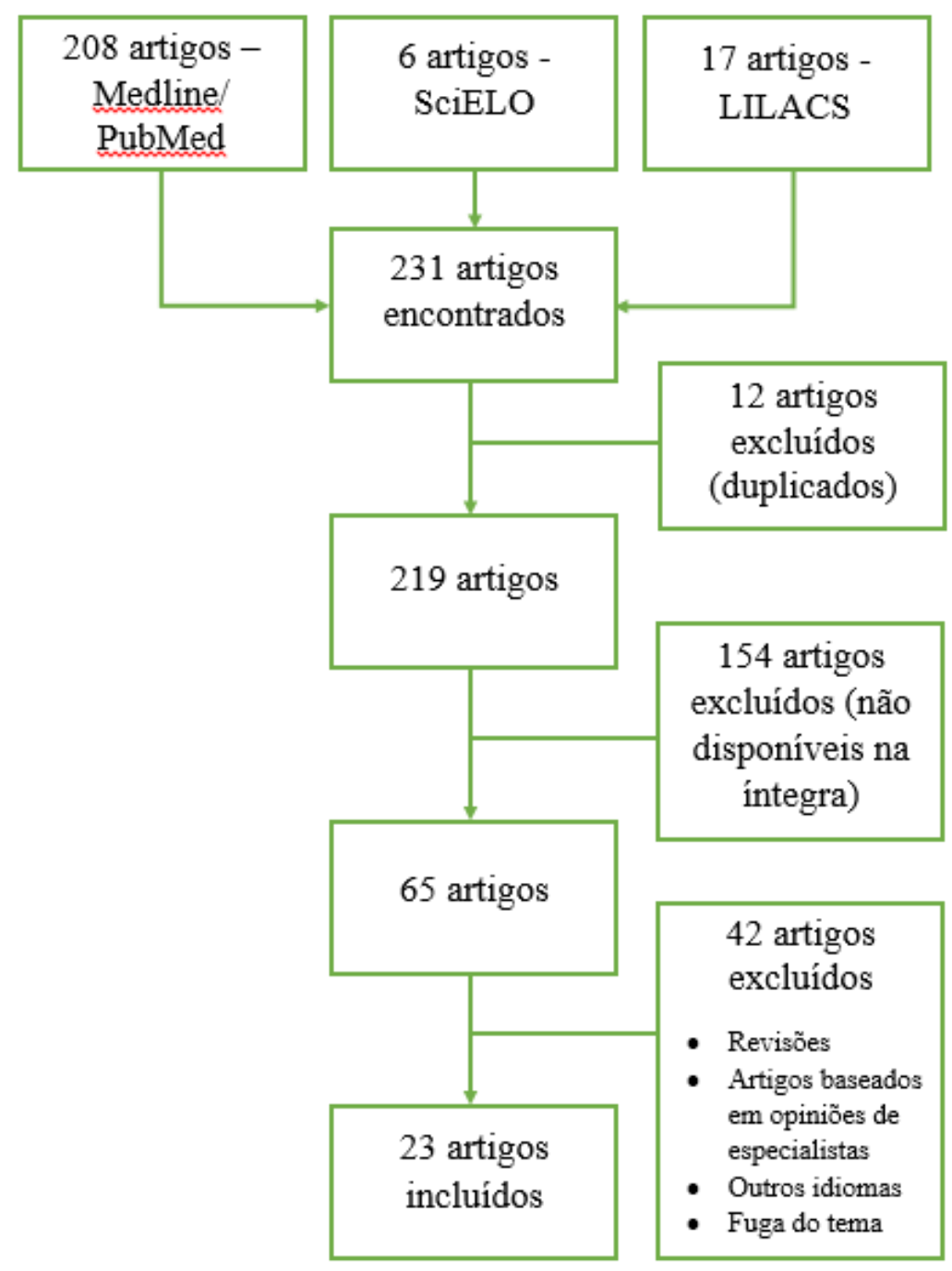

Fonte: Barbosa et al. (2021).

Dessa maneira, foram criteriosamente incluídos 23 artigos na presente revisão, os quais foram tabulados no software Microsoft Excel® 2016, considerando autoria, ano de publicação, objetivo, número de participantes, tratamento e principais achados.

\section{Resultados e Discussão}

Os artigos incluídos na revisão foram sumarizados no Quadro 1, sendo identificados os autores, ano de publicação, $\mathrm{n}^{\circ}$ de participantes, objetivo, tratamento avaliado e os principais achados. 
Research, Society and Development, v. 10, n. 5, e35310514874, 2021

(CC BY 4.0) | ISSN 2525-3409 | DOI: http://dx.doi.org/10.33448/rsd-v10i5.14874

Quadro 1 - Artigos incluídos na revisão. Parnaíba, 2020.

\begin{tabular}{|c|c|c|c|c|}
\hline $\begin{array}{l}\text { Autores (ano de } \\
\text { publicação) }\end{array}$ & $\begin{array}{c}\mathbf{N}^{\circ} \text { de } \\
\text { participantes }\end{array}$ & Objetivos & Tratamento avaliado & Principais achados \\
\hline $\begin{array}{l}\text { Abadchi, Naeini e } \\
\text { Beheshtian (2019) }\end{array}$ & 40 & $\begin{array}{c}\text { Comparar a eficácia do laser de } \mathrm{CO}_{2} \text { fracionado } \\
\text { associado à terapia tópica com a terapia tópica } \\
\text { sozinha. }\end{array}$ & $\begin{array}{l}\text { Laser de } \mathrm{CO}_{2} \text { fracionado } \\
\text { associado a hidroquinona } \\
\text { tópica } 4 \% \text {. }\end{array}$ & $\begin{array}{c}\text { Redução significativa na pigmentação foi observada após } 3 \text { semanas de } \\
\text { terapia combinada e } 6 \text { semanas após monoterapia. Foi observada redução } \\
\text { na homogeneidade após } 6 \text { semanas de ambos os tratamentos. Os } \\
\text { tratamentos não foram considerados significativamente diferentes em } \\
\text { nenhuma sessão ou pela utilização dos escores }(p>0,05) \text {. }\end{array}$ \\
\hline Arrowitz et al. (2019) & 59 & $\begin{array}{c}\text { Avaliar a eficácia e tolerância de formulação } \\
\text { contendo tiamidol 0,2\% e compará-la com } \\
\text { formulação de hidroquinona } 2 \% \text {, em mulheres com } \\
\text { melasma moderado. }\end{array}$ & $\begin{array}{l}\text { Formulação com tiamidol } \\
\qquad, 2 \%\end{array}$ & $\begin{array}{c}\text { Após } 12 \text { semanas, as pontuações do mMASI melhoraram } \\
\text { significativamente nos lados da face tratados com tiamidol } 0,2 \% \text { e } \\
\text { hidroquinona } 2 \% \text {. Durante o tratamento, nenhum sujeito apresentou piora } \\
\text { nas pontuações do mMASI no lado tratado com tiamidol, enquanto } \\
\text { aproximadamente } 10 \% \text { dos sujeitos mostraram uma piora no mMASI no } \\
\text { lado tratado com hidroquinona. }\end{array}$ \\
\hline Guo et al. (2019) & 12 & $\begin{array}{l}\text { Explorar as vantagens, eficácia e segurança do laser } \\
\text { QS Nd:YAG de } 1064 \text { nm no tratamento de melasma, } \\
\text { em pacientes chineses. }\end{array}$ & $\begin{array}{l}\text { Laser QS Nd:YAG de } 1064 \\
\text { nm em modo quickly-pulse- } \\
\text { to-pulse e modo de pulso } \\
\text { único }\end{array}$ & $\begin{array}{c}\text { Ambos os lados da face de cada paciente mostraram efeito terapêutico } \\
\text { semelhante. A experiência de menor dor e a reação de eritema cutâneo } \\
\text { mais leve foram relatados no lado do tratamento com o modo quickly- } \\
\text { pulse-to-pulse, em comparação ao modo de pulso único. }\end{array}$ \\
\hline $\begin{array}{l}\text { Jiang, Akinseye, Tovar- } \\
\text { Garza e Pandya (2018) }\end{array}$ & 6 & $\begin{array}{l}\text { Entrevistar seis pacientes diagnosticados com } \\
\text { melasma sobre o efeito da doença na sua qualidade } \\
\text { de vida e autoestima antes e depois do tratamento. }\end{array}$ & $\begin{array}{l}\text { Ácido tranexâmico e creme } \\
\text { de combinação tripla } \\
\text { (hidroquinona, retinol e } \\
\text { esteroide tópico). }\end{array}$ & $\begin{array}{l}\text { Todas as pacientes relataram efeitos negativos significativos na qualidade } \\
\text { de vida e autoestima, bem como melhora acentuada na autoestima após } \\
\text { tratamento com uma combinação tripla de creme e ácido tranexâmico oral. }\end{array}$ \\
\hline $\begin{array}{c}\text { Akabane, Almeida e Simão } \\
(2017)\end{array}$ & 9 & $\begin{array}{l}\text { Avaliar a eficácia, em relação à qualidade de vida e à } \\
\text { melhora objetiva, do uso de Polypodium leucotomos } \\
\text { no tratamento do melasma. }\end{array}$ & $\begin{array}{l}\text { Comprimido de } 250 \mathrm{mg} \mathrm{de} \\
\text { Polypodium leucotomos. }\end{array}$ & $\begin{array}{l}\text { Houve melhora do MASI em 55,6\% das pacientes após } 45 \text { dias de } \\
\text { tratamento. A despeito da discreta melhora no MASI, houve reflexo na } \\
\text { melhora dos escores de qualidade de vida (DLQI e MELASQoL). }\end{array}$ \\
\hline $\begin{array}{l}\text { Alavi, Abolhasani, Asadi e } \\
\text { Nilforoushzadeh (2017) }\end{array}$ & 41 & $\begin{array}{c}\text { Avaliar e comparar a combinação do laser Nd: YAG } \\
\text { Q-switch (QSNY) e laser fracionário de érbio: YAG } \\
\text { (FEYL) com laser QSNY sozinho no tratamento do } \\
\text { melasma. }\end{array}$ & Lasers QSNY e FEYL & $\begin{array}{l}\text { A cor da pele tornou-se mais clara em ambos os grupos com resultados } \\
\text { significativamente melhores no grupo que combinou QSNYL e FEYL } \\
\text { (QSNYL-FEYL). A porcentagem de diminuição do conteúdo de melanina } \\
\text { foi significativamente maior no grupo QSNYL-FEYL. A intensidade do } \\
\text { eritema pós-tratamento foi significativamente menor no grupo QSNYL- } \\
\text { FEYL. As pacientes não relataram eventos adversos. }\end{array}$ \\
\hline $\begin{array}{l}\text { Atefi, Dalvand, Ghassemi, } \\
\text { Mehran e Heydarian (2017) }\end{array}$ & 60 & $\begin{array}{c}\text { Avaliar e comparar os efeitos terapêuticos benéficos } \\
\text { e efeitos colaterais do ácido tranexâmico tópico em } \\
\text { comparação com hidroquinona no tratamento de } \\
\text { mulheres com melasma. }\end{array}$ & Ácido tranexâmico 5\% tópico & $\begin{array}{l}\text { A pontuação média do MASI em ambos os grupos de tratamento diminuiu } \\
\text { consideravelmente após a conclusão do tratamento e não foi } \\
\text { significativamente diferente entre os dois grupos. Em relação ao nível de } \\
\text { satisfação do paciente, as pacientes que fizeram uso do ácido tranexâmico }\end{array}$ \\
\hline
\end{tabular}


Research, Society and Development, v. 10, n. 5, e35310514874, 2021

(CC BY 4.0) | ISSN 2525-3409 | DOI: http://dx.doi.org/10.33448/rsd-v10i5.14874

\begin{tabular}{|c|c|c|c|c|}
\hline & & & & $\begin{array}{l}\text { tiveram um nível de satisfação significativamente maior, de } 33,3 \% \text {, em } \\
\text { comparação com } 6,7 \% \text { no grupo das pacientes que usaram hidroquinona. }\end{array}$ \\
\hline $\begin{array}{l}\text { Faghihi, Taheri, } \\
\text { Shahmoradi e } \\
\text { Nilforoushzadeh (2017) }\end{array}$ & 42 & $\begin{array}{c}\text { Avaliar a eficácia e segurança de solução com ácido } \\
\text { azelaico, resorcinol e ácido fítico no peeling químico } \\
\text { no tratamento de melasma em comparação com ácido } \\
\text { glicólico 50\%. }\end{array}$ & $\begin{array}{l}\text { Solução de ácido azelaico, } \\
\text { resorcinol e ácido fítico }\end{array}$ & $\begin{array}{l}\text { Os pacientes apresentaram melhora acentuada calculada com o escore } \\
\text { MASI antes e depois do tratamento em ambos lados da face. A eficácia da } \\
\text { fórmula combinada (ácido azelaico, resorcinol e ácido fítico) foi } \\
\text { semelhante ao ácido glicólico, mas com menos complicações. Não houve } \\
\text { diferença significativa com relação à melhora entre os dois grupos. }\end{array}$ \\
\hline $\begin{array}{l}\text { Lima, Lima, Paixão e Miot } \\
\text { (2017) }\end{array}$ & 6 & $\begin{array}{l}\text { Investigar alterações clínicas e histológicas } \\
\text { promovidas pelo microagulhamento no melasma } \\
\text { facial. }\end{array}$ & $\begin{array}{l}\text { Microagulhamento e } \\
\text { aplicação de combinação } \\
\text { tripla }\end{array}$ & $\begin{array}{l}\text { Foi percebida melhora do melasma em todas as pacientes. Houve redução } \\
\text { significativa dos escores MASI e MELASQoL. Todos os casos } \\
\text { evidenciaram espessamento do epitélio, diminuição da pigmentação da } \\
\text { melanina e densificação do colágeno da derme superior. As pacientes } \\
\text { foram acompanhadas por } 6 \text { meses sem recidiva. }\end{array}$ \\
\hline XU et al. (2017) & 28 & $\begin{array}{l}\text { Avaliar a eficácia de um microagulhamento } \\
\text { combinado com ácido tranexâmico tópico para } \\
\text { melasma em mulheres de meia idade na China. }\end{array}$ & $\begin{array}{l}\text { Microagulhamento com ácido } \\
\text { tranexâmico }\end{array}$ & $\begin{array}{l}\text { O escore Visia foi significativamente menor no tratamento com a terapia } \\
\text { combinada após } 12 \text { semanas. Os escores de satisfação subjetiva em ambos } \\
\text { os lados aumentaram significativamente. Os participantes ficaram mais } \\
\text { satisfeitos com os resultados do lado da terapia combinada do que do lado } \\
\text { do controle. }\end{array}$ \\
\hline Chen et al. (2016) & 23 & $\begin{array}{l}\text { Avaliar o resultado após o tratamento combinado } \\
\text { com sonoforese de vitamina } \mathrm{C} \text { e laser Nd:YAG, em } \\
\text { casos selecionados de hiperpigmentação facial. }\end{array}$ & $\begin{array}{l}\text { Laser Nd:YAG seguido por } \\
\text { sonoforese de vitamina C }\end{array}$ & $\begin{array}{l}\text { Em ambos os desfechos objetivos ou subjetivos, } 91,3 \% \text { dos pacientes } \\
\text { apresentaram resultado excelente ou melhor, enquanto } 8,7 \% \text { não } \\
\text { apresentaram alteração. A maioria dos pacientes (73,9\%) não apresentou } \\
\text { hiperpigmentação pós-inflamatória ou ligeira hiperpigmentação pós- } \\
\text { inflamatória que rapidamente se resolveu em } 1 \text { semana. Apenas um }(4,3 \%) \\
\text { paciente apresentou hiperpigmentação pós-inflamatória extrema que durou } \\
\text { mais de um mês. }\end{array}$ \\
\hline $\begin{array}{l}\text { Eshghi, Khezrian e Ashari } \\
\qquad(2016)\end{array}$ & 42 & $\begin{array}{c}\text { Estudar a eficácia da utilização de esteroides tópicos } \\
\text { na diminuição da pigmentação da pele, no tratamento } \\
\text { do melasma, comparando com a Fórmula Kligman. }\end{array}$ & $\begin{array}{l}\text { Microinjeções } \\
\text { subepidérmicas de } \\
\text { triancinolona }\end{array}$ & $\begin{array}{l}\text { A diminuição no MASI foi observada em ambos os grupos. Contudo, foi } \\
\text { significativamente maior no grupo que recebeu as injeções } \\
\text { subepidérmicas. }\end{array}$ \\
\hline Kim et al. (2016) & 22 & $\begin{array}{l}\text { Investigar a eficácia e efeitos adversos após poucas } \\
\text { sessões de Laser Q-comutado Nd:YAG 1064nm no } \\
\text { modo de pulso duplo fotoacústico em mulheres } \\
\text { asiáticas com melasma. }\end{array}$ & $\begin{array}{l}\text { Laser Q-comutado Nd:YAG } \\
\text { 1064nm no modo de pulso } \\
\text { duplo fotoacústico }\end{array}$ & $\begin{array}{l}\text { A avaliação geral dos investigadores e dos pacientes indicou melhora } \\
\text { significativa para } 59,1 \% \text { e } 86,4 \% \text { dos pacientes, respectivamente. As } \\
\text { pontuações MASI foram significativamente reduzidas em } 20,4 \% \text {. A } \\
\text { luminosidade, medida com um colorímetro, aumentou significativamente } \\
\text { em } 1,3 \text { ponto. Não foram observados eventos adversos notáveis. }\end{array}$ \\
\hline Medeiros et al. (2016) & 1 & $\begin{array}{c}\text { Apresentar os resultados de um caso clínico em que } \\
\text { se utilizou tratamento combinado a uma paciente com } \\
\text { melasma e relatar a eficácia das combinações } \\
\text { terapêuticas em comparação à monoterapia no } \\
\text { tratamento dessa dermatose. }\end{array}$ & $\begin{array}{c}\text { Terapia combinada (peeling } \\
\text { oral com ácido tranexâmico, } \\
\text { antioxidantes, fitoterápicos, } \\
\text { minerais e tópicos). }\end{array}$ & $\begin{array}{c}\text { Obteve-se notável melhora no clareamento do melasma ao exame da pele e } \\
\text { na autoestima, segundo relato da paciente. }\end{array}$ \\
\hline
\end{tabular}


Research, Society and Development, v. 10, n. 5, e35310514874, 2021

(CC BY 4.0) | ISSN 2525-3409 | DOI: http://dx.doi.org/10.33448/rsd-v10i5.14874

\begin{tabular}{|c|c|c|c|c|}
\hline $\begin{array}{l}\text { Adalatkhah e Sadeghi- } \\
\text { Bazargani (2015) }\end{array}$ & 74 & $\begin{array}{l}\text { Investigar a eficácia do creme de flutamida } 1 \% \text { em } \\
\text { comparação com o creme de hidroquinona } 4 \% \text { no } \\
\text { melasma. }\end{array}$ & Creme de flutamida $1 \%$ & $\begin{array}{l}\text { A flutamida tópica pareceu tão eficaz quanto a hidroquinona tópica no } \\
\text { tratamento do melasma usando avaliação com mexameter, mas com uma } \\
\text { tendência de melhora do MASI e maior satisfação do paciente no } \\
\text { tratamento com flutamida em comparação com a hidroquinona tópica. }\end{array}$ \\
\hline Lima (2015) & 18 & $\begin{array}{c}\text { Avaliar o tratamento do melasma recalcitrante com o } \\
\text { microagulhamento. }\end{array}$ & $\begin{array}{l}\text { Microagulhamento seguido } \\
\text { por fórmula de } \\
\text { despigmentação (tretinoína, } \\
\text { hidroquinona e } \\
\text { fluorocinolona acetonida) }\end{array}$ & $\begin{array}{c}\text { Todos os pacientes reportaram satisfação com o tratamento. Os resultados } \\
\text { foram considerados bons ou muito bons, de acordo com escala que } \\
\text { compreende: muito bom, bom, razoável e ruim. }\end{array}$ \\
\hline $\begin{array}{l}\text { Zhong, Sun, Liu, Niu e Wu } \\
\text { (2015) }\end{array}$ & 31 & $\begin{array}{l}\text { Avaliar a eficácia e segurança da lignina peroxidase } \\
\text { (LIP) como agente clareador da pele em pacientes } \\
\text { com melasma. }\end{array}$ & $\begin{array}{l}\text { Loção contendo lignina } \\
\text { peroxidase }\end{array}$ & $\begin{array}{l}\text { A loção clareadora LIP foi capaz de reduzir significativamente a } \\
\text { pigmentação da pele. A pontuação do MASI diminuiu estatisticamente } \\
\text { após } 28 \text { dias de tratamento. Nenhum evento adverso relacionado ao } \\
\text { tratamento foi observado. }\end{array}$ \\
\hline Ebrahimi e Naeini (2014) & 50 & $\begin{array}{c}\text { Avaliar a eficácia e segurança da solução tópica de } \\
\text { ácido tranexâmico 3\% e compará-la com a solução } \\
\text { combinada de hidroquinona 3\% e dexametasona } \\
0,01 \% \text { como o tratamento para melasma em mulheres } \\
\text { iranianas. }\end{array}$ & $\begin{array}{l}\text { Solução tópica de ácido } \\
\text { tranexâmico } 3 \%\end{array}$ & $\begin{array}{l}\text { Uma tendência decrescente significativa foi observada no escore MASI de } \\
\text { ambos os grupos, sem diferença significativa entre eles durante o estudo. } \\
\text { No entanto, os efeitos colaterais de hidroquinona com dexametasona foram } \\
\text { significativamente proeminentes em comparação com ácido tranexâmico. }\end{array}$ \\
\hline Diosti et al. (2012) & 10 & $\begin{array}{c}\text { Avaliar a eficácia do laser de érbio:YAG fracionado, } \\
\text { analisar histologicamente as características usuais do } \\
\text { melasma e a quantidade de pigmento na epiderme e } \\
\text { derme antes e após o tratamento. }\end{array}$ & $\begin{array}{l}\text { Laser de érbio:YAG 2940nm } \\
\text { fracionado }\end{array}$ & $\begin{array}{c}\text { Não foi observada melhora do escore MASI ao longo do tratamento. Em } \\
\text { sete casos observou-se redução no grau de hiperpigmentação da epiderme, } \\
\text { sem significância estatística. Portanto, o tratamento do melasma com o } \\
\text { laser de érbio: YAG fracionado ablativo não se mostrou efetivo, apesar de } \\
\text { haver tendência a diminuição dos escore MASI e no grau de } \\
\text { hiperpigmentação. }\end{array}$ \\
\hline $\begin{array}{l}\text { Iraji, Tagmirriahi e } \\
\text { Gavidnia (2012) }\end{array}$ & 72 & $\begin{array}{l}\text { Comparar a eficácia da solução de sulfato de zinco } \\
10 \% \text { com creme de hidroquinona } 4 \% \text { na melhora do } \\
\text { melasma. }\end{array}$ & $\begin{array}{l}\text { Solução de sulfato de zinco } \\
\qquad 10 \%\end{array}$ & $\begin{array}{c}\text { Após } 2 \text { meses de tratamento houve redução significativa nos escores } \\
\text { MASI de ambos os grupos. No entanto, o grupo que utilizou a } \\
\text { hidroquinona apresentou maiores diminuições do escore. Além disso, os } \\
\text { pacientes do grupo da hidroquinona continuaram a diminuir o escore } \\
\text { MASI pelo restante do período do estudo em } 6 \text { meses de } \\
\text { acompanhamento, ao passo que os pacientes do grupo do sulfato de zinco } \\
\text { não apresentaram continuada redução no escore MAIS após os dois meses } \\
\text { iniciais. }\end{array}$ \\
\hline Costa et al. (2010) & 56 & $\begin{array}{c}\text { Avaliar a eficácia e segurança clínicas do complexo } \\
\text { despigmentante emblica, licorice e belides, em } \\
\text { comparação à hidroquinona } 2 \%, \text { na abordagem do } \\
\text { melasma. }\end{array}$ & $\begin{array}{l}\text { Creme à base de complexo } \\
\text { despigmentante emblica, } \\
\text { licorice e belides } 7 \%\end{array}$ & $\begin{array}{c}\text { Não houve diferença estatística na melhora do melasma entre os grupos. } \\
\text { Contudo, o grupo que utilizou o creme à base de complexo despigmentante } \\
\text { apresentou menor incidência de eventos adversos. }\end{array}$ \\
\hline
\end{tabular}


Research, Society and Development, v. 10, n. 5, e35310514874, 2021

(CC BY 4.0) | ISSN 2525-3409 | DOI: http://dx.doi.org/10.33448/rsd-v10i5.14874

\begin{tabular}{|c|c|c|c|c|}
\hline Magalhães et al. (2010) & 33 & $\begin{array}{c}\text { Avaliar, através do MASI e MELASQoL, o efeito do } \\
\text { peeling de ácido láctico em pacientes portadores de } \\
\text { melasma }\end{array}$ & $\begin{array}{c}\text { Peelings seriados de ácido } \\
\text { láctico } 85 \%\end{array}$ & $\begin{array}{c}\text { Observou-se melhora significativa de ambos os índices, ou seja, houve } \\
\text { melhora clínica do melasma além de melhora na qualidade de vida das } \\
\text { pacientes após o tratamento com o peeling de ácido láctico. Entretanto, não } \\
\text { houve correlação significativa entre os escores das escalas MASI e } \\
\text { MELASQoL tanto no início quanto no final do tratamento, constatando-se } \\
\text { que a melhora clínica nem sempre corresponde ao grau de expectativa das } \\
\text { pacientes. }\end{array}$ \\
\hline $\begin{array}{l}\text { Moreira, Bravo, Amorim, } \\
\text { Luiz e Issa (2010) }\end{array}$ & 13 & $\begin{array}{c}\text { Avaliar a eficácia e segurança do Skin Whitening } \\
\text { Complex (SWC) no tratamento do melasma facial, e } \\
\text { compará-lo à hidroquinona. }\end{array}$ & $\begin{array}{l}\text { SWC: complexo } \\
\text { despigmentante com extrato } \\
\text { de uva-ursina, Aspergillus } \\
\text { biofermentado, extrato de } \\
\text { grapefruit e extrato de arroz. }\end{array}$ & $\begin{array}{c}\text { Das } 13 \text { pacientes, } 10 \text { apresentaram melhora clínica global do melasma. Na } \\
\text { hemiface tratada com hidroquinona } 4 \% \text {, a melhora foi total em } 4 \text { pacientes, } \\
\text { e parcial em } 6 \text {. Na hemiface tratada com SWC 5\%, a melhora foi total em } \\
2 \text { pacientes, e parcial em } 6 \text {. Embora o tratamento com hidroquinona } 4 \% \\
\text { apresente melhor resultado clínico, não houve diferença estatisticamente } \\
\text { significativa entre os dois tratamentos }\end{array}$ \\
\hline
\end{tabular}

Fonte: Barbosa et al. (2021) 
De acordo com os tratamentos empregados nos trabalhos selecionados, foram criadas 5 categorias para serem discutidas na revisão: tratamentos utilizando ácido tranexâmico, tratamentos com microagulhamento, tratamentos com laser, tratamentos com extratos vegetais e outros tratamentos.

\section{Tratamentos utilizando ácido tranexâmico}

O uso de ácido tranexâmico no tratamento de melasma moderado a grave vem sendo recentemente indicado por sua eficácia terapêutica alcançada com poucos efeitos colaterais (Jiang et al., 2018). O ácido age proporcionando a diminuição da melanogênese em melanócitos epidérmicos e o clareamento rápido e sustentado das manchas (Ayuthaya, Niumphradit, Manosroi, \& Nakakes, 2012).

Atefi et al. (2017) compararam, em um estudo duplo-cego randomizado com 60 pacientes, tratamentos realizados com ácido tranexâmico e hidroquinona. As pacientes foram divididas em dois grupos, o primeiro foi tratado com ácido tranexâmico 5\% tópico duas vezes ao dia por 12 semanas, o segundo grupo foi tratado com hidroquinona 2\% nas mesmas condições. As áreas foram avaliadas antes da intervenção e após o tratamento quanto ao escurecimento e extensão com base na sua área e o índice MASI. A pontuação MASI diminuiu em ambos os grupos, porém sem diferença significativa. Entretanto, quanto aos efeitos colaterais, foram maiores as reclamações no segundo grupo, incluindo eritema e irritação local, além de uma maior satisfação do primeiro grupo comparado com o outro grupo. O uso tópico do ácido diminuiu a pigmentação por melanina e a pontuação MASI, constituindo-se em tratamento eficaz com poucos efeitos indesejáveis e grande satisfação das pacientes, quando comparado com o uso de hidroquinona, sendo recomendado o seu uso.

Jiang et al. (2018) utilizaram uma terapia combinada de ácido tranexâmico oral $325 \mathrm{mg}$ duas vezes ao dia, triplo creme de combinação composto por $6 \%$ de hidroquinona, $0,0125 \%$ de tretinoína e $0,1 \%$ de dexametasona, utilizado uma vez ao dia, além do uso de proteção solar, em seis mulheres, duas com melasma considerado grave e quatro com melasma moderado. Todas elas experimentavam sentimentos negativos em relação à condição. As pacientes do estudo já vinham de tratamentos anteriores com creme de hidroquinona a 4\%, creme de tretinoína e luz pulsada intensa, por no mínimo três meses, sem melhora. Após o tratamento instituído, relataram melhora da qualidade de vida, apresentando maior disposição a participar de atividades sociais, ao ar livre e com maior autoconfiança. O pequeno número de participantes e a natureza qualitativa do estudo, porém, tornam os resultados pouco generalizáveis.

Em seu estudo, Xu et al. (2017) compararam o uso de microagulhamento (microarray funcional) combinado com ácido tranexâmico tópico 0,5\% uma vez por semana, por 12 semanas, em tratamento de melasma em 28 mulheres de meia idade chinesas, obtendo resultados positivos de melhora e satisfação no uso combinado em comparação com a realização apenas do microagulhamento, e recomendando a utilização dessa terapêutica, uma vez que o microagulhamento pode contribuir para o aumento da eficácia do ácido, é uma terapia segura, indolor e sem grandes efeitos colaterais.

Medeiros et al. (2016) também citam o uso de ácido tranexâmico como alternativa no tratamento da hipercromia. O ácido tem sido utilizado como peeling sistêmico; também por meio de microinjecção intradérmica (uso mais eficaz que apenas o tópico), mostrando-se terapia efetiva e promissora. Outra opção é a administração por via oral (250 mg, 12/12 horas) apresentando, da mesma forma, bons resultados com poucos efeitos adversos, essa opção também pode ser combinada de maneira segura com laser de baixa frequência.

Ebrahimi e Naeini (2014) corroboram os achados destas pesquisas ao comparar a terapêutica utilizando ácido tranexâmico tópico $3 \%$ com o uso combinado de hidroquinona $3 \%$ e dexametasona $0,01 \%$ num estudo duplo-cego com 50 mulheres iranianas com melasma. Apesar de haver resultados semelhantes quanto ao clareamento e à satisfação com ambos os tratamentos, os efeitos colaterais com hidroquinona e dexametasona foram significativamente maiores, sendo a terapêutica 
com ácido tranexâmico indicada por se tratar de um medicamento seguro e eficaz.

Sendo assim, os estudos atestam a eficácia do uso de ácido tranexâmico no tratamento do melasma, principalmente pela segurança do seu uso, sem efeitos colaterais significativos, entretanto, afirmam a necessidade da realização de novas pesquisas para determinar os mecanismos do ácido tranexâmico nesse tratamento, definir a sua frequência de uso, benefícios e malefícios a longo prazo e uso de terapias combinadas (Ebrahimi \& Naeini, 2014; Atefi et al., 2017; Xu et al., 2017).

\section{Tratamentos com microagulhamentos}

Lima et al. (2017) avaliaram a realização de duas sessões de microagulhamento (Dr. Roller®, $1.5 \mathrm{~mm}$ ) a cada 30 dias (T0 e T30), seguido (no dia seguinte) pela aplicação de combinação tripla diária - hidroquinona, fluocinolona e tretinoína (Tri-Luma, Galderma®) e protetor solar de amplo espectro (Anthelios Airlicium SPF 70 com cor, da La Roche Posay®) para o tratamento de melasma refratário. Foi considerado melasma refratário aquele com mais de cinco anos de evolução e recaídas depois de três tentativas de tratamento, incluindo combinação tripla.

Outro autor que também utilizou-se do microagulhamento mais combinação tripla para tratamento do melasma refratário foi Lima (2015). Ele considerou como melanose refratária aquelas que não respondiam ao clareamento tópico e uso de protetor solar. O tratamento empregado nesta pesquisa foi o uso de microagulhas (Dr.Roller ${ }^{\circledR}$ 1,5mm) - com lidocaína creme a $4 \%$ trinta minutos antes da intervenção para anestesia local - unido a fórmula despigmentante 24 horas depois do procedimento e nos dias seguintes (tretinoína $0,05 \%$ + hidroquinona $4 \%$ + de acetoneto de fluocinolona $1 \%$ ) e protetor solar com cor e FPS 60. O mesmo procedimento foi realizado 30 dias após o primeiro tratamento, ou seja, uma abordagem semelhante à utilizada por Lima et al. (2017). O trauma causado no procedimento deve ser modesto e torna-se obrigatório o uso de clareador de pele (fórmula despigmentante) e protetor solar após o procedimento. Como resultado, Lima et al., (2017) obtiveram redução de aproximadamente $70 \%$ no MASI, aumento de $13 \%$ na luminância da pele e queda de $55 \%$ no MELASQoL. Além disso, uma análise histológica comparativa em amostra de pele antes e depois do tratamento evidenciou, no pós-tratamento, espessamento do epitélio, diminuição da pigmentação melânica epitelial e diminuição da densificação do colágeno da derme superior. A membrana basal, a qual foi danificada no melasma, mostrou traços de restauração após o tratamento. Tais participantes do estudo foram acompanhados, ainda, por 6 meses com uso de protetor solar de amplo espectro e combinação tripla sem apresentar recaída.

Já na pesquisa de Lima (2015), os métodos para avaliar a eficácia da intervenção foram outros, mas também apresentaram resultados positivos. Foram observados resultados de bons a muito bons em uma escala de muito bom; bom; razoável e pobre apresentada pelos autores. Estes também concluíram que todos os 22 pacientes foram responsivos à técnica usada e que, com isso, pretendem repetir o mesmo procedimento em outros casos com indicação semelhante. Do total de pacientes, 55\% já estavam em acompanhamento há 24 meses após o primeiro procedimento (no momento da publicação) e estavam mantendo clareamento cutâneo semelhante ao observado há dois meses depois da primeira intervenção.

Com isso, a união do microagulhamento de pele com o tratamento clássico (filtro solar de amplo espectro e combinação tripla) promoveu melhora clínica e histológica do melasma facial refratário. Mais estudos randomizados controlados são necessários agora para investigar qual o melhor regime de microagulhamentos, a fim de maximizar sua eficácia no tratamento da melanose refratária (Lima et al., 2017).

Outro tratamento que se utilizou do microagulhamento foi retratado por Xu et al. (2017) o qual comparou a utilização do microarray funcional de microagulhas unido ao uso do ácido tranexâmico tópico 0,5\%, uma vez por semana durante 12 semanas em uma hemiface, versus o uso de um dispositivo como simulação do microarray funcional unido a solução tópica de ácido tranexâmico $0,5 \%$, pelo mesmo período de tempo, na outra metade do rosto. O microarray funcional de microagulhas é 
uma tecnologia de um cartucho com nanochip que contém nanopontas, o que permite a penetração de tais microagulhas na pele com uma frequência de 3000 vezes por minuto. Seu objetivo é facilitar a absorção transdérmica de ácido tranexâmico tópico (Xu et al., 2017). Como resultado, observou-se uma melhor satisfação do paciente no lado do rosto tratado, também, com a técnica de microagulhas, por meio de um score de satisfação criada pelos autores. Em relação à pigmentação da pele, não houve diferença significativa ao se comparar o lado estudado ao lado controle. Entretanto, ao se observar as manchas mais pigmentadas, estas foram menos encontradas no lado que utilizou a técnica de microagulhas. O índice de melanina também foi medido e, após a semana 12 de tratamento, este foi significativamente menor no tratamento com o emprego de microagulhas. Em suma, este estudo descobriu que o ácido tranexâmico tópico 0,5\% aplicado posterior ao pré-tratamento com microarray de microagulhas produziu efeitos significativamente melhores sobre o melasma em comparação ao ácido tranexâmico tópico sozinho após 12 semanas de terapia. Além disso, o pré-tratamento com microarray funcional de microagulhas permitiu que todo o processo fosse indolor, sendo muito mais aceitável do que as injeções locais de ácido tranexâmico normalmente utilizadas (Xu et al., 2017).

Por tudo isso, o tratamento com microagulhamento associado a solução despigmentante é eficaz no tratamento do melasma, inclusive nos casos refratários, portanto deve ser considerado na abordagem terapêutica da melanose.

\section{Tratamentos com laser}

Inúmeras são as possibilidades de terapia quando o assunto é o tratamento do melasma. Entretanto, apesar dessa ampla gama terapêutica, o manejo a longo prazo dessa condição ainda é um desafio. Em busca de alternativas para garantir melhores resultados no tratamento dessa doença, alguns estudos discutem a eficácia do uso de lasers em pacientes com melasma refratário (Diosti et al., 2012).

Diosti et al. (2012) avaliou a eficácia do laser de érbio:YAG fracionado individualmente e após três sessões, através do escore MASI, da análise subjetiva e da análise histológica nos dez participantes de seu estudo. Esse tratamento foi bem tolerado pelos participantes e considerado seguro, no entanto, observou-se que o escore MASI não apresentou diminuição estatisticamente significativa (Diosti et al., 2012).

Em contraponto, ao analisar subjetivamente esses resultados os participantes relataram melhora dos seus quadros de melasma refratário. Em suma, a pequena amostra desse estudo sugere a melhora clínica e histológica proporcionada pelo laser de érbio, entretanto, o tratamento apresentou uma grande quantidade de recidivas, evidenciando os desafios para o manejo a longo prazo dessa doença (Diosti et al., 2012).

Chen et al. (2016) realizou um estudo com 23 mulheres buscando avaliar a eficácia do laser Nd:YAG como terapêutica para atenuar a hiperpigmentação facial, dentre elas, 4 pacientes já haviam realizado tratamento anterior com o laser de érbio, obtendo uma fraca resposta. Diante disso, o essa modalidade de laser foi utilizada buscando obter melhores resultados no tratamento da hiperpigmentação. Assim, o uso do laser Nd:YAG quando associado à sonoforese de vitamina C foi descrito como uma alternativa eficaz no manejo dessa problemática, pois apresentou uma melhora significativa e uma diminuição na taxa de recidivas a longo prazo (Chen et al., 2016).

Nesse estudo, foram realizadas cinco sessões do laser em combinação com sonoforese de vitamina C imediatamente após o primeiro procedimento. Essa associação apresentou benefícios significativos na diminuição dos casos de recidivas, na resolução dos casos de hiperpigmentação pós-inflamatória severa e, ainda, na maior duração dos efeitos do tratamento (Chen et al., 2016). Ainda segundo os autores, a aplicação subsequente da vitamina C por sonoforese possibilita uma maior absorção da mesma, pois o laser destrói de maneira reversível a barreira cutânea. Essa melhora associada à sinergia das duas terapêuticas no tratamento do melasma foi confirmada por Kim et al. (2016). No entanto, faz-se necessário um projeto de estudo 
prospectivo randomizado controlado para provar os efeitos específicos dessa sinergia (Chen et al., 2016).

Nessa perspectiva, Kim et al. (2016) destacou em seu estudo a eficácia da tonificação a laser para obtenção de resultados significativos no tratamento do melasma sem qualquer ônus para os pacientes. Isso se dá uma vez que a nova geração de lasers Nd:YAG utiliza a tecnologia Q-comutado PTP que emite um feixe de energia duplo localizado, minimizando os danos térmicos. Essa tecnologia possibilita minimizar os efeitos colaterais indesejados da terapia a laser, como a hiperpigmentação pós-inflamatória descrita anteriormente (Kim et al., 2016).

Entretanto, existem algumas limitações dessa modalidade de tratamento, como o alto número de sessões para a obtenção de uma melhora clínica satisfatória. Apesar disso, o tratamento foi bem aceito pelas pacientes e considerado seguro por não apresentar efeitos colaterais importantes. Por fim, nesse estudo não foi avaliado o grau de recorrência, sendo uma necessidade importante para posterior avaliação dessa terapêutica como eficaz no tratamento a longo prazo do melasma (Kim et al., 2016).

Uma outra modalidade de protocolo de tratamento do melasma consiste na associação de diferentes tipos de lasers, como proposto por Alavi et al. (2017). Em seu estudo foram associados o laser ablativo fracionário de érbio:YAG (FEYL) e o laser não ablativo Q-switch Nd:YAG (QSNYL), sendo o grupo controle composto por pacientes que receberam apenas o tratamento com QSNYL. Cabe ressaltar ainda, que outras medidas além das técnicas envolvendo laser foram utilizadas, como uso, por pelo menos uma semana antecedendo a sessão do laser, de fórmula Klingman $(0,1 \%$ de tretinóina, $5 \%$ de hidroquinona e $0,1 \%$ de dexametasona hidrofílica) a noite e administração de lidocaína $5 \%$ trinta minutos antes do procedimento com laser (Alavi et al., 2017). Como resultado, os dois grupos (QSNYL-FEYL e apenas QSNYL) obtiveram melhora estatisticamente significativa. Contudo, o grupo que recebeu ambas modalidades de lasers mostrou uma redução significativamente $(\mathrm{p}<0,001)$ maior do conteúdo de melanina quando comparado ao grupo QSNYL.

Outro laser que apresentou resultados significativos para o tratamento do melasma foi o laser QS Nd:YAG de 1064 nanômetros. Guo et al. (2019) avaliaram dois modos desse laser, o modo de pulso único e o modo Q-PTP (Quickly-pulse-topulse), modo que envolve pulsos com intervalos de 80 microssegundos, os quais alcançam uma maior energia acumulada, de forma mais segura que o laser de pulso único. Foram realizadas sessões dos dois modos do laser em cada paciente, cada modo em uma hemiface. $\mathrm{O}$ resultado encontrado foi a redução significativa da pigmentação do melasma nas pacientes, contudo, sem diferença estatística considerando o modo de laser empregado. No entanto, é importante salientar que o modo Q-PTP mostrouse mais indolor e com menores reações de eritema (Guo et al., 2019).

Abadchi et al. (2019) compararam a eficácia do laser de CO2 fracionado associado a terapia tópica com hidroquinona $4 \%$ à terapia tópica isolada. As sessões de laser foram realizadas com intervalos de 3 semanas. E a hidroquinona $4 \%$ foi utilizada em todo o rosto (lado controle e lado tratamento), duas vezes ao dia, a partir do terceiro dia após a sessão com laser. Foi observado que, embora resultados significativos de melhora do quadro de melasma tenham surgido mais precocemente (após 3 semanas) no grupo tratamento (laser + hidroquinona 4\%), o lado controle também apresentou melhora significativa (após 6 semanas), não havendo diferenças significativas entre os dois grupos. Sendo assim, é importante ressaltar que, a terapia com laser associada à hidroquinona tem a vantagem de apresentar resultados positivos mais precoces. Entretanto, uma vez que os resultados não apresentam diferença significativa, além do fato da terapia a laser ser de alto custo, não há razão para indicar a terapia associada, considerando o longo prazo (Abadchi et al., 2019).

Dessa forma, diferentes tipos de lasers, sejam eles ablativos ou não ablativos, ou ainda em seus diferentes modos de pulso, podem ser utilizados com bons resultados no tratamento do melasma (Chen et al., 2016; Abadchi et al., 2019). Contudo, deve-se ponderar quando o emprego de tal técnica é justificado frente a utilização de outros tratamentos tópicos já bem consolidados (Kim et al., 2016; Abadchi et al., 2019). 


\section{Tratamentos com extratos vegetais}

Em estudo realizado por Costa et al. (2010) foram comparados os efeitos da hidroquinona $2 \%$ e de formulação contendo os agentes vegetais emblica, licorice e belides a 7\% no tratamento do melasma. Nesse estudo, um grupo utilizou a hidroquinona uma vez à noite e o outro, os agentes vegetais duas vezes ao dia, levando à melhora clínica e ausência da piora das lesões em ambos. Contudo, nenhum dos grupos relatou remissão do melasma, apenas a melhora das lesões. Os efeitos adversos relatados por aquelas que usaram extratos vegetais foram ardência e aumento do número de lesões acneicas prévias, já no grupo da hidroquinona, foram relatados eritema, ardência e pápulas eritematosas na região perioral (Costa et al., 2010).

Moreira et al. (2010), por sua vez, compararam a ação do Skin Whitenig Complex (SWC), um complexo composto por extrato de uva-ursina, um extrato vegetal, Aspergillus biofermentado, extrato de grapefruit e extrato de arroz, com a hidroquinona $4 \%$ na terapêutica do melasma, de forma duplo cega, com tubos de gel-creme a serem utilizados em cada hemiface. As pacientes de ambos os grupos durante o estudo não poderiam usar nenhum medicamento associado ao tratamento, apenas fazer uso de protetor solar fator 60. Não foram observadas diferenças significativas entre os dois tipos de manejo. Contudo, no tratamento que envolveu extrato de semente da uva-ursina não foram observados efeitos colaterais, ao contrário da hidroquinona, sendo o SWC uma opção terapêutica para o melasma principalmente em pacientes que tenham contraindicação ao uso de hidroquinona (Moreira et al., 2010).

Akabane et al. (2017) avaliaram o efeito de Polypodium leucotomos (PL) em mulheres com melasma. As participantes ingeriram comprimidos de $250 \mathrm{~g}$ de PL a cada 12 horas, por 45 dias, sendo observada redução significativa no MELASQoL e DLQI, bem como uma melhora do MASI em 55\% das participantes.

A utilização de substâncias derivadas de extratos vegetais é uma importante alternativa à utilização dos fármacos considerados padrões no tratamento do melasma. Tais substâncias, extraídas de porções distintas de diferentes vegetais, a exemplo da belides, da licorice, da emblica e da uva-ursina, derivados de flores, raízes, frutos e folhas, respectivamente, apresentaram efeitos positivos e semelhantes ao tratamento considerado padrão-ouro para o melasma, a hidroquinona (Costa et al., 2010; Moreira et al., 2010).

\section{Outros tratamentos}

Além dos tratamentos que puderam ser agrupados nas seções anteriores, os artigos selecionados apontaram ainda outras terapêuticas que serão contempladas adiante.

Faghihi et al. (2017) avaliaram a aplicação de peeling usando uma combinação que consistiu de 20\% de ácido azeláico, $10 \%$ de resorcinol e 6\% de ácido fítico, comparando-o com aplicação de ácido glicólico 50\% e obtiveram como resultados a redução dos escores MASI para aproximadamente metade dos valores iniciais para os dois tratamentos empregados, não havendo diferença estatística significativa entre os grupos. Dessa forma, a combinação tripla de ácido azelaico, resorcinol e ácido fítico tem eficácia similar ao peeling de ácido glicólico no tratamento para melasma (Faghihi et al., 2017).

Outro tratamento envolvendo peeling recuperado na seleção de artigos foi o peeling de ácido lático 85\%, estudado por Magalhães et al. (2010), os quais procederam com sessões seriadas da técnica em 33 pacientes com melasma. Foi observada redução nos índices MASI e MELASQoL, quando comparados ao momento inicial da pesquisa, sem associação significativa entre os índices, percebendo-se que nem sempre a melhora clínica é compatível com as expectativas das pacientes.

Zhong et al. (2015) avaliaram a utilização da lignina peroxidase (LP) tópica, uma enzima obtida do fungo Phanerochaete chrysosporium, capaz de quebrar a lignina - molécula parecida com a melanina -, no tratamento do melasma. A utilização da LP aumentou significativamente os parâmetros de luminosidade em comparação com a utilização apenas de 
protetor solar. Foi ainda observada diminuição significativa da vermelhidão prévia nas áreas com melasma.

Chama-se atenção ao fato dos trabalhos de Magalhães et al. (2010) e Zhong et al. (2015) não compararem o peeling de ácido lático $85 \%$ e a utilização da LP, respectivamente, com nenhum tratamento de referência, resultando em uma lacuna no conhecimento se esses tratamentos são mais ou menos eficazes no manejo do melasma que os tratamentos normalmente já empregados.

Iraji et al. (2012) avaliaram a utilização de solução de sulfato de zinco $10 \%$ comparando-a à hidroquinona $4 \%$. A hidroquinona, como já esperado, por ser um tratamento de referência no melasma, mostrou diminuição do MASI ao longo dos seis meses de estudo. Contudo, a solução de sulfato de zinco 10\%, apesar de resultar em leve diminuição no escore MASI nos primeiros 2 meses, não resultou em diferenças significativas ao longo dos 6 meses de estudo. Dessa forma, os pesquisadores concluem a pesquisa ressaltando que o sulfato de zinco tópico não foi um tratamento efetivo para o melasma (Iraji et al. 2012).

Adalatkhah e Sadeghi-Bazargani (2015) avaliaram a eficácia de flutamida tópica $1 \%$ comparada à hidroquinona $4 \%$ no tratamento de melasma. A flutamida é um agente antiandrogênico normalmente utilizado no tratamento do carcinoma prostático, mas que também tem sido proposto o uso em situações como acne, hirsutismo, queda de cabelo e melasma (Adalatkhah \& Sadeghi-Bazargani, 2015; Muramatsu et al., 2019). Nos dois grupos avaliados houve melhora da hiperpigmentação, não havendo diferença estatística significativa nas medições de melanina entre os dois grupos usando a avaliação com Mexameter, após 4 meses. Contudo, comparado ao grupo controle, a satisfação das pacientes que utilizaram flutamida foi significativamente maior.

A aplicação de triamcinolona intralesional, um corticosteróide, também é um dos tratamentos estudados para o manejo do melasma. Eshghi et al. (2016) compararam a triamcinolona intralesional à utilização da fórmula Kligman, a qual contém hidroquinona $5 \%$, tretinoína $0,1 \%$ e dexametasona $0,1 \%$. Os pesquisadores obtiveram como resultado a redução estatisticamente significativa do escore MASI em ambos tratamentos, considerando os escores iniciais. Na comparação entre os grupos foi observada uma eficácia superior da triamcinolona intralesional, uma vez que os escores MASI desse grupo foram estatisticamente inferiores $(\mathrm{p}<0,001)$ ao que utilizou a fórmula Kligman (Eshghi et al., 2016). Contudo, como desvantagem desse procedimento, tem-se a queixa de dor no momento da aplicação, o que, de acordo com os autores, não foi considerado um empecilho, quando explicados os possíveis benefícios da técnica.

Arrowitz et al. (2019) estudaram a aplicação do tiamidol em manchas de melasma. O tiamidol, ou isobutylamidothiazolyl-resorcinol, é um potente inibidor da tirosinase humana, tendo ação in vitro superior a hidroquinona, arbutina e ácido kójico (Mann et al., 2018; Arrowitz et al., 2019). O estudo comparou a aplicação de formulação de 0,2\% de tiamidol com formulação de hidroquinona $2 \%$ e com a aplicação apenas de protetor solar. Os pesquisadores observaram que o uso de tiamidol e de hidroquinona por 12 semanas resultaram em diminuições significativas no mMASI nos dois grupos de mulheres, quando comparados ao controle (protetor solar). Contudo, as pacientes tratadas com tiamidol experimentaram uma redução ainda maior $(\mathrm{p}<0,001)$, quando comparadas àquelas tratadas com hidroquinona.

\section{Conclusão}

O manejo do melasma é um desafio para a medicina visto que seu quadro muitas vezes é refratário e seu tratamento é de longa duração, além de ser capaz de apresentar variações de efeitos adversos - a depender do método terapêutico de escolha. Este artigo considerou que ao comparar o tratamento tópico com ácido tranexâmico e hidroquinona os resultados são semelhantes, no entanto a segunda opção apresenta mais efeitos adversos. Somado a isso, verifica-se que em pacientes que apresentam recidivas frequentes a associação do microagulhamento contribui com aumento da eficácia do ácido tranexâmico, 
Research, Society and Development, v. 10, n. 5, e35310514874, 2021

(CC BY 4.0) | ISSN 2525-3409 | DOI: http://dx.doi.org/10.33448/rsd-v10i5.14874

sendo a terapia com laser outra opção de manejo nesses casos. Ademais, as formulações contendo extratos vegetais, com os agentes emblica, licorice, belides e da uva-ursina apresentam melhora das manchas hipercrômicas semelhantes a ação da hidroquinona, assim como outros tratamentos à base flutamida tópica, triamcinolona intralesional e tiamidol. Já em relação ao sulfato de zinco, pesquisadores concluíram que este não é um tratamento efetivo para o melasma, mas que a combinação tripla de ácido azelaico, resorcinol e ácido fítico tem eficácia similar ao peeling de ácido glicólico. Contudo, estes estudos são considerados insuficientes dado que não os compara com nenhum tratamento de referência. Dessa forma, fazem-se necessários novos estudos que abordem o tratamento do melasma, de forma a encontrar novas opções terapêuticas com resultados de longa duração e reduzidos efeitos colaterais.

\section{Referências}

Abadchi, S. N., Naeini, F. F., \& Beheshtian, E. (2019). Combination of hydroquinone and fractional CO2 laser versus hydroquin one monotherapy in melasma treatment: A randomized, single-blinded, split-face clinical trial. Indian journal of dermatology, 64(2), 129.

Adalatkhah, H., \& Sadeghi-Bazargani, H. (2015). The first clinical experience on efficacy of topical flutamide on melasma compared with topical hydroquinone: a randomized clinical trial. Drug design, development and therapy, 9, 4219.

Akabane, A. L., Almeida, I. P., \& Simão, J. C. L. (2017). Avaliação dos índices de qualidade de vida (MELASQoL e DLQI) e do MASI em pacientes com melasma tratadas com Polypodium Leucotomos. Surgical \& Cosmetic Dermatology, 9(3), 214-217.

Alavi, S., Abolhasani, E., Asadi, S., \& Nilforoushzadeh, M. (2017). Combination of Q-switched Nd: YAG and fractional Erbium: YAG lasers in treatment of melasma: a randomized controlled clinical trial. Journal of lasers in medical sciences, $8(1), 1$.

Arrowitz, C., Schoelermann, A. M., Mann, T., Jiang, L. I., Weber, T., \& Kolbe, L. (2019). Effective tyrosinase inhibition by thiamidol results in significant improvement of mild to moderate melasma. Journal of Investigative Dermatology, 139(8), 1691-1698.

Atefi, N., Dalvand, B., Ghassemi, M., Mehran, G., \& Heydarian, A. (2017). Therapeutic effects of topical tranexamic acid in comparison with hydroquinone in treatment of women with melasma. Dermatology and therapy, 7(3), 417-424.

Ayuthaya, P. K. N., Niumphradit, N., Manosroi, A., \& Nakakes, A. (2012). Topical 5\% tranexamic acid for the treatment of melasma in Asians: a double-blind randomized controlled clinical trial. Journal of Cosmetic and Laser Therapy, 14(3), 150-154.

Chen, Y. T., Chang, C. C., Hsu, C. R., Shen, J. H., Shih, C. J., \& Lin, B. S. (2016). Combined vitamin C sonophoresis and neodymium-doped yttrium aluminum garnet (NdYAG) laser for facial hyperpigmentation: An outcome observation study in Asian patients. Indian Journal of Dermatology, Venereology, and Leprology, 82(5), 587.

Costa, A., Moisés, T. A., Cordero, T., Alves, C. R. T., \& Marmirori, J. (2010). Associação de emblica, licorice e belides como alternativa à hidroquinona no tratamento clínico do melasma. Anais Brasileiros de Dermatologia, 85(5), 613-620.

Costa, F. V., Souza, L. S. de, Conradesque, P. R., \& Carniel, P. (2020). Therapeutic response of cysteamine in the treatment of melasma. Research, Society and Development, 9(6), e75963468.

Diosti, G. M., Mulinari-Brenner, F., Filus Neto, J., Werner, B., Nascimento, A., \& Piva, F. M. (2012). Avaliação clínica e histológica de pacientes com melasma refratário tratadas com laser de érbio: Yag fracionado. Surgical \& cosmetic dermatology, 4(2), 114-120.

Ebrahimi, B., \& Naeini, F. F. (2014). Topical tranexamic acid as a promising treatment for melasma. Journal of research in medical sciences: the official journal of Isfahan University of Medical Sciences, 19(8), 753.

Eshghi, G., Khezrian, L., \& Esna Ashari, F. (2016). Comparison between Intralesional Triamcinolone and Kligman's Formula in Treatment of Melasma. Acta medica Iranica, 54(1), 67-71.

Faghihi, G., Taheri, A., Shahmoradi, Z., \& Nilforoushzadeh, M. A. (2017). Solution of azelaic acid (20\%), resorcinol (10\%) and phytic acid (6\%) versus glycolic acid (50\%) peeling agent in the treatment of female patients with facial melasma. Advanced Biomedical Research, 6.

Garcia, A. K. A., Fonseca, L. F., Aroni, P., \& Galvão, C. M. (2016). Estratégias para o alívio da sede: revisão integrativa da literatura. Revista Brasileira de Enfermagem, 69(6), 1148-55.

Guo, X., Cai, X., Jin, Y., Zhang, T., Wang, B., \& Li, Q. (2019). Q-PTP is an optimized technology of 1064-nm Q-switched neodymium-doped yttrium aluminum garnet laser in the laser therapy of melasma: A prospective split-face study. Oncology letters, 18(4), 4136-4143.

Hammerschmidt, M., Mattos, S. M. L., Suzuki, H. S., Freitas, C. F. N. P., \& Mukai, M. M. (2012). Avaliação dos métodos de classificação do melasma de acordo com a resposta ao tratamento. Surgical \& Cosmetic Dermatology, 4(2), 155-158.

Iraji, F., Tagmirriahi, N., \& Gavidnia, K. (2012). Comparison between the efficacy of $10 \%$ zinc sulfate solution with $4 \%$ hydroquinone cream on improvement of melasma. Advanced Biomedical Research, 1. 
Research, Society and Development, v. 10, n. 5, e35310514874, 2021

(CC BY 4.0) | ISSN 2525-3409 | DOI: http://dx.doi.org/10.33448/rsd-v10i5.14874

Jiang, J., Akinseye, O., Tovar-Garza, A., \& Pandya, A. G. (2018). The effect of melasma on self-esteem: A pilot study. International journal of women's dermatology, 4(1), 38-42.

Kim, J. Y., Choi, M., Nam, C. H., Kim, J. S., Kim, M. H., Park, B. C., \& Hong, S. P. (2016). Treatment of melasma with the photoacoustic twin pulse mode of low-fluence 1,064 nm Q-switched Nd: YAG laser. Annals of dermatology, 28(3), 290-296.

Lima, E. D. A. (2015). Microneedling in facial recalcitrant melasma: report of a series of 22 cases. Anais brasileiros de dermatologia, 90(6), 919-921.

Lima, E. V., Lima, M. M. D., Paixão, M. P., \& Miot, H. A. (2017). Assessment of the effects of skin microneedling as adjuvant therapy for facial melasma: a pilot study. BMC dermatology, 17(1), 1-6.

Magalhães, G. M., Borges, M. D. F. M., Vieira, P. J., \& Neves, D. R. (2010). Peeling de ácido láctico no tratamento do melasma: avaliação clínica e impacto na qualidade de vida. Surgical \& Cosmetic Dermatology, 2(3), 173-179.

Mann, T., Gerwat, W., Batzer, J., Eggers, K., Scherner, C., Wenck, H., Stäb, F., Hearing, V. J., Röhm, K. H., \& Kolbe, L. (2018). Inhibition of human tyrosinase requires molecular motifs distinctively different from mushroom tyrosinase. Journal of Investigative Dermatology, 138(7), 1601-1608.

Medeiros, J. K. G., Neves, W. W., Moura, N. M. D., \& Medina, W. S. G. (2016). Combinação terapêutica no tratamento do melasma. CuidArte, Enferm, 180187.

Moreira, A. M., Bravo, B. S. F., Amorim, A. G. F., Luiz, R. R., \& Issa, M. C. A. (2010). Estudo duplo cego comparativo entre hidroquinona e extrato de uvaursina no tratamento do melasma. Surgical \& Cosmetic Dermatology, 2(2), 99-104.

Muramatsu, T., Funahashi, Y., Yamamoto, A., Sassa, N., Matsukawa, Y., \& Gotoh, M. (2019). A case of advanced prostate cancer controlled for the long term by flutamide after bicalutamide failure. Nagoya journal of medical science, 81(4), 707-710.

Oliveira, A. A., Gonçalves, P. F., Santos, K. S., Duarte, S. F. P., David, I. R., \& Santos, J. A. B. (2019). Impacto do Melasma na Autoestima de Mulheres/Impact of Melasma on Women's Self-Estems. ID on line REVISTA DE PSICOLOGIA, 13(48), 435-443.

Souza, M. T., Silva, M. D., \& Carvalho, R. (2010). Revisão integrativa: o que é e como fazer. Einstein, 8(1), 102-106.

Xu, Y., Ma, R., Juliandri, J., Wang, X., Xu, B., Wang, D., Lu, Y., Zhou, B., \& Luo, D. (2017). Efficacy of functional microarray of microneedles combined with topical tranexamic acid for melasma: A randomized, self-controlled, split-face study. Medicine, 96(19).

Zhong, S. M., Sun, N., Liu, H. X., Niu, Y. Q., \& Wu, Y. (2015). Reduction of facial pigmentation of melasma by topical lignin peroxidase: a novel fast-acting skin-lightening agent. Experimental and therapeutic medicine, 9(2), 341-344. 